\title{
Rostromedial Septal Area Controls Pulsatile Growth Hormone Release in the Golden Hamster
}

\author{
KATARINA T. BORER \\ Department of Kinesiology, The University of Michigan, 401 Washtenaw, Ann Arbor, MI 48109
}

Received 20 October 1986

\begin{abstract}
BORER, K. T. Rostromedial septal area controls pulsatile growth hormone release in the golden hamster. BRAIN RES BULL 18(4) 485-490, 1987. - Limbic forebrain inhibits growth and growth hormone (GH) secretion in mature golden hamsters as shown by acceleration of growth and increases in serum $\mathrm{GH}$ concentrations following the electrolytic lesions of septum, transection of the hippocampus and surgical separation of these two regions. The growth-inhibitory function of this circuit is most probably mediated by somatostatinergic (SRIF) neurons. Such lesions induce hypoactivity possibly due to damage to endogenous opiatergic (EOP) neurons. EOP neurons facilitate spontaneous running in hamsters and mediate exercise-induced acceleration of growth and GH pulses. The coincidence of hypoactivity and growth acceleration after such lesions suggested the coexistence of SRIF and EOP fibers within the growth-inhibitory limbic forebrain circuit which control the rate of growth in mature hamsters by the variable inhibition of SRIF neurons by the EOP neurons. This hypothesis posits that accelerated growth is due to increased GH pulse frequency, and hypoactivity due to damage to EOP neurons, and was tested in this study by mcasuring pulsatile GH release (and as a measure of specificity, pulsatile prolactin release) in the presence and in the absence of opiate-receptor blocker naloxone in 21 female hamsters which sustained electrocoagulative lesions of rostromedial septum and 30 hamsters subjected to control surgery. Lesions doubled GH but not PRL pulse frequency, neither of which was affected by naloxone. Results support the hypothesis that opiatergic neurons facilitate pulsatile GH release by inhibiting the action of somatostatin neurons.
\end{abstract}

Septum Somatostatin Endogenous opiates Prolactin Growth

A limbic forebrain circuit inhibits growth hormone $(\mathrm{GH})$ release and somatic growth in mature golden hamsters. It consists of fibers interconnecting the hypothalamus and hippocampus which traverse rostromedial septum $[5,6,9,12]$. The growth and GH-inhibitory function of this circuit in the mature hamster is most probably mediated by somatostatinergic fibers [11]. Thus damage to this limbic forebrain circuit in the rostromedial septum, in dorsal hippocampus, or the surgical separation of these two regions, is accompanied by facilitation of $\mathrm{GH}$ release and striking acceleration of somatic and skeletal growth $[5,6,9,12]$. Growth-inducing transection of dorsal hippocampus results in depletion of somatostatin (SRIF) in the hippocampus and accumulation of SRIF in septum proximal to the cut, suggesting interruption of fibers originating in septum or anterior hypothalamus and innervating hippocampus [11]. On the other hand, administration of antisomatostatin serum increases $G H$ pulse frequency and amplitude in neurologically intact adult hamsters [8] and provides further evidence for the involvement of this peptide in the suppression of $\mathrm{GH}$ secretion in adult hamsters.

Several lines of evidence suggest that endogenous opiates (EOP) within the limbic forebrain facilitate $\mathrm{GH}$ release in the hamster. Thus EOPs appear to sustain high levels of voluntary running in hamsters [27]. Such exercise facilitates GH release [2] and accelerates somatic and skeletal growth in hamsters [2,7]. Exercise-induced EOP release mediates facilitation of GH release in mature hamsters through inhibition of endogenous somatostatin action [8].

Damage to the growth-inhibitory limbic circuit in mature hamsters leads to striking hypoactivity $[5,9,10]$, just as opiate-receptor blockade induces hypoactivity in neurologically-intact hamsters [27]. The coincidence of hypoactivity and growth acceleration following the damage to the hypothalamo-hippocampal circuit in mature hamsters suggested the coexistence and anatomical proximity of opiatergic fibers within the growth-inhibitory limbic forebrain circuit. Thus damage to somatostatinergic fibers [11] within this circuit would account for the observed acceleration of somatic growth and increased serum GH concentrations $[5,6,9]$, and damage to the opiatergic circuit would account for the observed hypoactivity of these animals $[5,9$, 10]. The hypothesis of proximity of somatostatinergic and opiatergic fibers thus helps explain both the observed interaction between EOP and SRIF neurons in the neurologically intact exercising hamsters [8] and the coincidence of growth acceleration and hypoactivity following damage to the growth-inhibitory limbic forebrain circuit $[5,9,10]$.

The purpose of the present experiment was to test the hypothesis that the growth rate in mature female golden hamsters is controlled by the variable inhibition of SRIF neurons by the EOP neurons within the growth-inhibitory limbic forebrain circuit. This hypothesis predicts that electrolytic lesons of the rostromedial septum would damage 
somatostatin neurons and remove the inhibition over pulsatile GH secretion. Furthermore, these lesions would make opiate inhibition of SRIF action ineffective.

\section{METHOD}

\section{Animals and Maintenance}

Female golden Syrian hamsters (Mesocricetus auratus Waterhouse) were obtained from Engle Laboratory Animals, Farmersburg, IN at the age of about 100 days and weighing 90 to $100 \mathrm{~g}$. Animals were individually housed in suspended metal cages with wire floors and supplied with water and Purina Formulab chow ad lib. Animals were maintained under controlled light (12 L:12 D) and temperature $\left(20-22^{\circ} \mathrm{C}\right)$ conditions.

\section{Surgical Procedures}

Electrocoagulative lesions were produced under sodium pentobarbital anesthesia ( $75 \mathrm{mg} / \mathrm{kg}$ IP). No. 1 insect pins, insulated with epoxylite except for $0.5 \mathrm{~mm}$ at the tip, served as electrodes. With the skull level between bregma and lambda, electrode was lowered $5 \mathrm{~mm}$ below the dura and 2 $\mathrm{mm}$ anterior to the bregma in the midline. In the experimental animals, direct anodal current $(2 \mathrm{~mA})$ was delivered for $20 \mathrm{sec}$. In control hamsters, the electrode was lowered only $3 \mathrm{~mm}$ below the dura and no current was delivered. Brains were fixed in $10 \%$ buffered formalin after removal from animals killed by an overdose of anesthetic and given a cardiac perfusion with saline and formalin solution. Alternate $80 \mu \mathrm{m}$ coronal sections were stained with cresyl violet and examined histologically to determine the location and the extent of damage. The extent of brain damage was outlined on diagrams of representative coronal sections of a hamster brain 161 .

\section{Blood Collection}

A chronic intracardiac polyethylene catheter (PE 50 , Intramedic) was implanted for a distance of $2 \mathrm{~cm}$ inside the right jugular vein in anesthetized hamsters (sodium pentobarbital, $80 \mathrm{mg} / \mathrm{kg}$ ). Catheter was kept patent with heparinized saline $(200 \mathrm{U} / \mathrm{ml})$ between blood collections to prevent blood-clot formation. A half of a $\mathrm{ml}$ of blood was collected every $20 \mathrm{~min}$ for a $6 \mathrm{hr}$ beginning at $0900 \mathrm{hr}$ and 24 hr after catheterization. Previous studies $[3,4]$ revealed no evidence of postoperative stress on pulsatile $\mathrm{GH}$ and prolactin (PRL) release 24 hours after intracardiac catheterization. Blood samples were packed by centrifugation at $4^{\circ} \mathrm{C}$, and plasma was kept at $-20^{\circ} \mathrm{C}$ for subsequent hormone analyses. Blood cells were washed twice in saline and twice in citrate phosphate dextrose buffer $[19,20]$. Blood was reconstituted with the same buffer $(50 \%)$ and human plasma protein fraction (Plasmanate, Cutter Laboratories, Berkeley, CA, 50\%). Reconstituted blood was reinfused into the animals in equal volumes following each blood removal.

\section{Hormone Assays}

Homologous radioimmunoassays were used to measure hamster GH [4] and hamster PRL [3]. PRL determinations served to check the specificity of lesion and drug effects on the pulsatile GH release. Purified hamster hormones, antibodies against hamster hormones, hamster PRL iodinated by the chloramine $T$ [3] and rat GH (NIADDK rat GH-I-3) iodinated by the lactoperoxidase procedure [34] were used in the assays. In the GH RIA, intrassay coeffi-

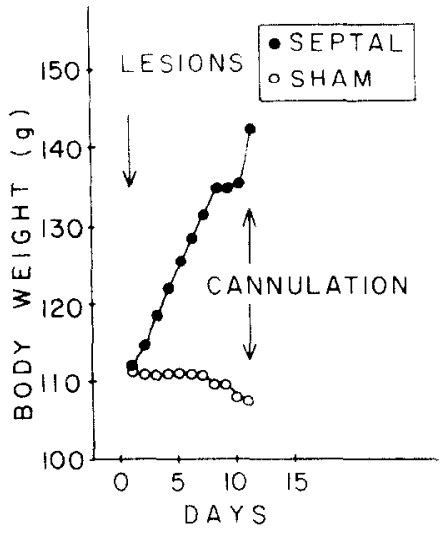

FIG. 1. Postoperative weight changes in 21 septal-lesioned hamsters (solid circles) and 30 sham-lesioned hamsters (open circles).

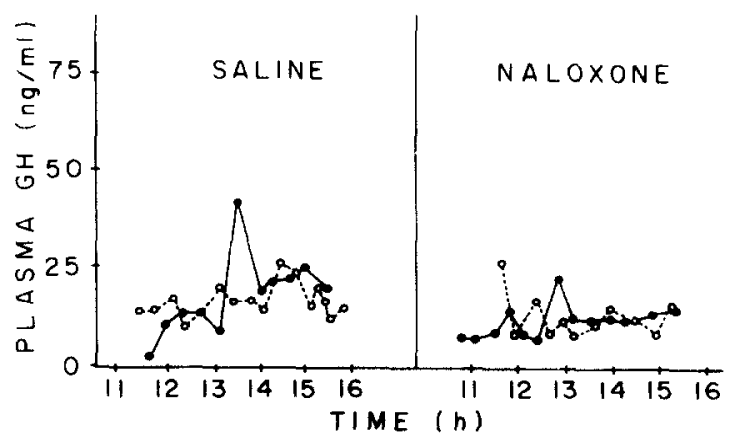

FIG. 2. Pulsatile pattern of GH secretion in two sham-lesioned hamsters injected with saline (left) and two sham-lesioned hamsters injected with naloxone (right).

cients of variation at $20 \%$ and $80 \%$ specific binding were $8.8 \%$ and $17.5 \%$ and interassay coefficient of variation for a pool of hamster standard serum was $16.8 \%$. Assay sensitivity ( 2 SD of buffer control tubes) was $596 \mathrm{pg} / \mathrm{ml}$. In the PRL assay, intraassay coefficients of variation were $5.7 \%$ and $13.3 \%$, interassay coefficient of variation of the hamster standard serum was $12.6 \%$, and assay sensitivity was 302 $\mathrm{pg} / \mathrm{ml}$. Of the 51 hamsters used in this study, both hormones were measured in the blood of 28 , and $\mathrm{GH}$ only in the blood of 23 .

\section{Drug Administration}

Naloxone $\mathrm{HCl}$ (E. I. Du Pont de Nemours \& Co., Wilmington, DE) at a dose of $20 \mathrm{mg} / \mathrm{kg}$ was dissolved in $2 \mathrm{ml}$ of saline and administered IP within five minutes of the collection of the first blood sample. Control animals received an IP injection of saline at the same time. 


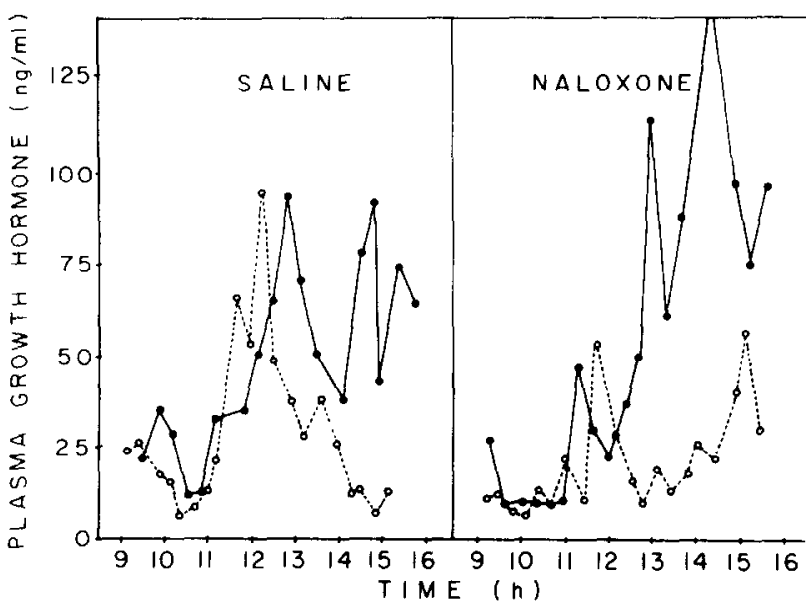

FIG. 3. Pulsatile pattern of GH secretion in two septal-lesioned hamsters injected with saline (left) and two septal-lesioned hamsters injected with naloxone (right).

\section{Experimental Design}

Twenty-one hamsters received septal lesions and 30 were sham operated. Two weeks after surgery, hamsters received intracardiac catheters $[3,4]$ and were housed individually in suspended metal cages with wire floors, similar to home cages, in the room in which blood collection took place $24 \mathrm{hr}$ later. Subsequent to connecting and flushing of the catheters with heparinized saline at $0830 \mathrm{hr}$ on the day of blood collection, handling of the animals was kept to a minimum depending on the ease of blood removal. No special arrangements were made to isolate the animals from environmental stimuli, but the animals generally appeared undisturbed and frequently slept or ate over the period of blood collection. Within the septal-lesioned and sham-operated groups, 9 and 13 hamsters, respectively, received naloxone injection, and the remainder were injected with saline.

\section{Statistical Analysis}

Growth rates were determined from the slope of the least squares linear regressions of weight changes as a function of time. The use of this measure as an index of skeletal growth was validated previously [7]. Hormone data analysis, including parallelism determinations, was performed by a computer program [16]. GH and PRL pulses, amplitudes, and troughs were defined in the following way [8]. Overlap between 1.5 SEMs of duplicate hormone determinations for two blood samples served as the criterion of reliability of differences between them with a significance level of $3.4 \%$. Basal hormone values were calculated from lowest plasma concentrations with overlapping SEMs. Plasma hormone concentrations showing no SEM overlap with basal hormone concentrations met the criterion of peak if the rise and fall in GH or PRL concentration occurred within 100 minutes. Amplitude of each peak was the difference in concentration between the peak and the preceding and following basal hormone concentrations.

Mean values for peaks, amplitudes, and the baseline for each animal were used as data in two way analysis of variance. Logarithmic transformation of the amplitude and basal hormone data was used to normalize their distribution. Student's $t$-test was used for weight comparisons.

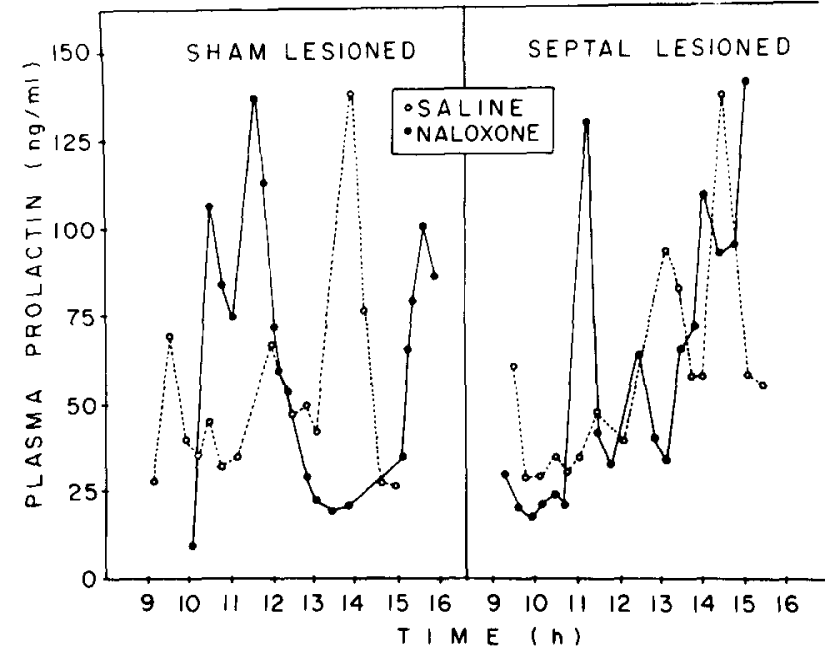

FIG. 4. Pulsatile pattern of PRL secretion in sham-lesioned hamsters (left) and septal-lesioned hamstcrs (right) which were injected with either saline (open circles) or naloxone (solid circles).

\section{RESULTS}

Septal lesions induced an eight-fold increase in the rate of growth ( $3.3 \pm 0.2$ vs. $-0.05 \pm 0.1 \mathrm{~g} /$ day, $p<0.001$, Fig. 1$)$.

Pulsatile hormone data are summarized in Table 1. Figure 2 presents representative pulsatile $\mathrm{GH}$ patterns in four different sham-operated hamsters injected with saline (left) or naloxone (right). Figure 3 presents corresponding data from four septal-lesioned hamsters, and Fig. 4, pulsatile PRL release in four representative animals that sustained sham lesions (left) or septal lesions (right) and which were injected with saline or naloxone. Septal lesions led to doubling of GH pulse frequency $(p<0.001)$ and to a $35 \%$ increase in $\mathrm{GH}$ pulse amplitude $(p<0.025$, Table 1 , and left sides of Figs. 2 and 3). Lesions had no effect on basal GH concentration nor on any aspect of pulsatile PRL release (Table 1 and Fig. 4).

Naloxone administration reduced $\mathrm{GH}$ pulse amplitude by $64 \%$ in sham-operated hamsters $(p<0.05$, Table 1, Fig. 2, right) and reduced baseline PRL concentration by $30 \%$ in both groups of hamsters $(p<0.025$, Table 1$)$. Naloxone administration had no effect on pulsatile GH release in septallesioned hamsters.

Lesions were predominantly located in the rostromedial septal area, anterior and dorsal to the anterior commissure (Fig. 5).

\section{DISCUSSION}

Our data are consistent with the hypothesis that a limbic forebrain circuit which interconnects hypothalamus and hippocampus, suppresses GH release and the rate of somatic growth in mature hamsters. Damage to this circuit through lesions of rostral septum [6], transection of septohippocampal connections [9], or transection of dorsal hippocampus [5], leads to oversecretion of $\mathrm{GH}$ and acceleration of somatic and skeletal growth in mature hamsters. In the present study we secured evidence that the inhibitory influence of this limbic circuit manifests itself through suppression of GH pulse frequency and amplitude. The effect is specific to $\mathrm{GH}$, as pulsatile PRL release remained unaffected. 
TABLE 1

EFFECTS OF SALINE OR NALOXONE ADMINISTRATION ON THE PULSATILE PATTERN OF GH AND PRL RELEASE IN SEPTAL-LESIONED AND SHAM-LESIONED HAMSTERS

\begin{tabular}{cccc}
\hline Groups & $\begin{array}{c}\text { Pulse } \\
\text { Frequency } \\
\text { (pulses/6 hr) }\end{array}$ & $\begin{array}{c}\text { Pulse } \\
\text { Amplitude } \\
\text { (ng/ml) }\end{array}$ & $\begin{array}{c}\text { Basal } \\
\text { Concentration } \\
\text { (ng/ml) }\end{array}$ \\
\hline
\end{tabular}

Growth Hormone

\begin{tabular}{|c|c|c|c|}
\hline $\begin{array}{l}\text { Septal lesion } \\
+ \text { saline }\end{array}$ & $3.5 \pm 0.2^{\text {a* }}$ & $51.5 \pm 13.2 \uparrow$ & $12.5 \pm 1.2$ \\
\hline $\begin{array}{c}\text { Sham lesion } \\
+ \text { saline }\end{array}$ & $1.8 \pm 0.4$ & $40.3 \pm 12.8$ & $12.7 \pm 1.0$ \\
\hline $\begin{array}{l}\text { Septal lesion } \\
+ \text { naloxone }\end{array}$ & $2.9 \pm 0.6^{*}$ & $36.4 \pm 10.7$ & $13.4 \pm 1.5$ \\
\hline $\begin{array}{l}\text { Sham lesion } \\
+ \text { naloxone }\end{array}$ & $1.8 \pm 0.4$ & $13.7 \pm 1.5 \ddagger$ & $12.4 \pm 1.0$ \\
\hline \multicolumn{4}{|c|}{ Prolactin } \\
\hline $\begin{array}{l}\text { Septal lesion } \\
+ \text { saline }\end{array}$ & $2.7 \pm 0.7$ & $47.7 \pm 9.8$ & $26.8 \pm 3.9$ \\
\hline $\begin{array}{c}\text { Sham lesion } \\
+ \text { saline }\end{array}$ & $3.1 \pm 0.3$ & $42.9 \pm 8.5$ & $25.8+3.9$ \\
\hline $\begin{array}{l}\text { Septal lesion } \\
+ \text { naloxone }\end{array}$ & $1.8 \pm 0.4$ & $43.7 \pm 12.1$ & $18.8 \pm 2.5 \dagger$ \\
\hline $\begin{array}{l}\text { Sham lesion } \\
+ \text { naloxone }\end{array}$ & $2.4 \pm 0.2$ & $63.5 \pm 13.5$ & $21.1 \pm 1.8^{+}$ \\
\hline
\end{tabular}

${ }^{3}$ Mean \pm SEM.

*Effect of septal lesion: $p<0.001$.

tEffect of septal lesion: $p<0.025$.

$\$$ Effect of naloxone in sham-lesioned hamsters: $p<0.05$.

Our research singles out somatostatin neurons as the most probable cause of suppression of $\mathrm{GH}$ pulse frequency and amplitude in mature golden hamsters. Thus immunoneutralization of circulating SRIF by antisomatostatin serum increased $\mathrm{GH}$ pulse frequency and amplitude in neurologically-intact hamsters to values that were higher than in hamsters induced to grow rapidly by exercise [8] or septal lesions (this experiment). Interruption of this fiber system by transection of dorsal hippocampus led to accumulation of somatostatin proximal to the cut and depletion of this neurotransmitter distal to the cut. The most probable site of origin of somatostatin fibers is the anterior and periventricular hypothalamus $[17,21]$ from where axons may traverse to hippocampus by way of rostromedial septum. Hippocampus by some as yet undefined route influences the activity of growth hormone releasing factor (GHRF) neurons in the medial basal hypothalamus [22] which constitute the final common path for the neural control of GH release in this species. Although most attention has been directed at the arcuate nucleus and the median eminence as the target areas of the anterior hypothalamic-periventricular somatostatin neurons $[14,15,17,18]$, innervation of limbic structures by somatostatin fibers has been previously demonstrated [25].

While opiate receptor blockade abolished exerciseinduced increases in GH pulse frequency and amplitude in neurologically intact hamsters [8] it has no effect on septallesion-induced increases in $\mathrm{GH}$ pulse frequency and amplitude (present study). Effectiveness of opiate-receptor block-

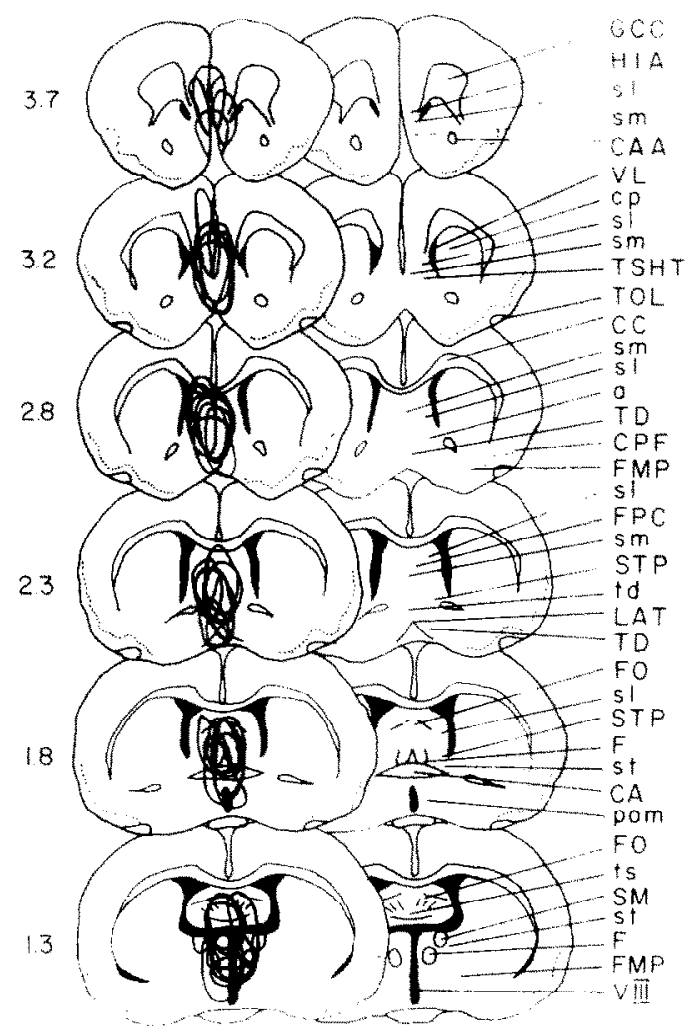

FIG. 5. Reconstruction of lesion damage in 21 hamsters which re ceived electrolytic lesions aimed at rostromedial septum. Numbers at left indicate distance of the section, in $\mathrm{mm}$, anterior to bregma. Abbreviations: a, nucleus accumbens; $\mathrm{CA}$, anterior commissure; $\mathrm{CAA}$, anterior limb of the anterior commissure; $\mathrm{CC}$, corpus callosum; $c p$, nucleus caudate putamen; CPF, pyriform cortex: F, column of the fornix; FMP, medial forebrain bundle; FO, fornix; FPC precommissural fornix; GCC, genu of corpus callosum; HIA, anterior part of hippocampus; LAT, lamina terminalis; pom, medial preoptic nucleus; sl, lateral septal nucleus; sm, medial septal nucleus; SM, stria medullaris; st, interstitial nucleus of stria terminalis; STP, precommissural part of stria terminalis; TD, tract of the diagonal band of Broca; td, nucleus of the diagonal band of Broca: TOL, lateral olfactory tract; $t$, triangular septal nucleus; TSHT, septohypothalamic tract; VL, lateral ventricle; VIII, third ventricle.

ade in restraining $\mathrm{GH}$ secretion in neurologically-intact exercising hamsters and its ineffectiveness in hamsters with limbic forebrain lesions supports the hypothesis of opiatergic inhibition of somatostatinergic action within the growthinhibitory limbic forebrain circuit in mature female hamsters. Thus the present and the preceding [8] studies support the notion that an opiatergic limbic circuit antagonizes the action of the hippocampal somatostatin. This circuit is activated by voluntary running activity [27], and the resultant EOP release has two biological effects. First, such EOP release sustains high levels of voluntary running [27], and the removal of this source of opiates following destruction of hypothalamo-hippocampal interconnections $[5,9,10]$ leads to profound hypoactivity. Second, this circuit restrains the action of hippocampal somatostatin $[8,11]$ and accelerates somatic growth. Damage to the somatostatinergic hypothalamo-hippocampal neurons accounts for increase in $\mathrm{GH}$ pulse frequency and amplitude. 
One can postulate that early rapid growth marks the phase of hamster development when somatostatinergic fibers have not developed or become active. Reduction in the spontaneous growth rate as the hamsters mature marks the development or activation of somatostatinergic circuit to hippocampus. Opiatergic fibers retain the capacity to restrain somatostatin action and thus permit variable degrees of somatic growth in mature hamsters.

Hamster provides a highly suitable model for the study of the neuroendocrine control of GH release and growth. One can noninvasively attenuate the inhibitory influence over growth and $\mathrm{GH}$ release in mature hamsters with voluntary running [2]. Anatomical location of the growth- and GHinhibitory circuit has been outlined within the limbic forebrain $[5,6,9]$. In addition, it appears that a relatively simple peptidergic mechanism modulates pulsatile GH release and the rate of growth in mature hamsters: SRIF maintains low GH pulsatility and slow rate of growth, and EOPs facilitate pulsatile $\mathrm{GH}$ release and accelerate growth by inhibiting SRIF action ([8] and this study).
Hamster model of the neuroendocrine control of growth offers several advantages over those available in other rodents such as rats and mice. First, hamster GH secretory response to stress shows greater similarity to primates $[13,23]$ and the human $[29,31]$ than to other rodents [30-33] which display stress-associated decrease in GH secretion. Next, there is a clear correspondence between the various measures of growth hormone release in the hamster and their rate of somatic growth $[2,5,6,9]$, while that is not the case in the rat $[24,28]$. And finally, neuroendocrine control mechanism for $\mathrm{GH}$ release is accessible to experimental analysis in the hamster, while the strong sexual dimorphism [28] of this mechanism in the rat complicates such analysis.

\section{ACKNOWLEDGEMENTS}

This research was supported in part by the National Science Foundation grants PCM 81-04375 and DCB 85-02902. Gift of naloxone by E. 1. Du Pont de Nemours \& Co. is gratefully acknowledged.

\section{REFERENCES}

1. Baker, B. L. and Y.-Y. Yu. Distribution of growth hormone release-inhibiting hormone (somatostatin) in the rat brain as observed with immunocytochemistry. Anat Rec 186: 343-356, 1976.

2. Borer, K. T. and R. P. Kelch. Increased serum growth hormone and somatic growth in exercising adult hamsters. Am $J$ Physiol 234: E611-E616, 1978

3. Borer, K. T., R. P. Kelch and K. Corley. Hamster prolactin: Physiological changes in blood and pituitary concentrations as measured by a homologous radioimmunoassay. Neuroendocrinology 35: 13-21, 1982.

4. Borer, K. T., R. P. Kelch and T. Hayashida. Hamster growth hormone: Species specificity and physiological changes in blood and pituitary concentrations as measured by a homologous radioimmunoassay. Neuroendocrinology 35: 349-358, 1982.

5. Borer, K. T., R. P. Kelch, J. Peugh and C. Huseman, Increased serum growth hormone and somatic growth in adult hamsters with hippocampal transections. Neuroendocrinology 29: 22-33, 1979.

6. Borer, K. T., R. P. Kelch, M. P. White, L. Dolson and L. R. Kuhns. The role of septal area in the neuroendocrine control of growth in the adult golden hamster. Neuroendocrinology 23: $133-150,1977$.

7. Borer, K. T. and L. R. Kuhns. Radiographic evidence for acceleration of skeletal growth in adult hamsters by exercise. Growth 41: $1-13,1977$.

8. Borer, K. T., D. R. Nicoski and V. Owens. Alteration of pulsatile growth hormone secretion by growth-inducing exercise: Involvement of endogenous opiates and somatostatin. Endocrinology 118: 844-850, 1986.

9. Borer, K. T., N. L. Peters, R. P. Kelch, A. C. Tsai and S. Holder. Contribution of growth, fatness, and activity to weight disturbance following septohypothalamic cuts in adult hamsters. J Comp Physiol Psychol 93: 907-918, 1979.

10. Borer, K. T., C. D. Potter and N. Fileccia. Basis for the hypoactivity that accompanies rapid weight gain in hamsters. Physiol Behav 30: 389-397, 1983.

11. Borer, K. T., B. Shapiro and A. I. Vinik. A role for somatostatin in the control of hamster growth. Brain Res Bull 11: 663-669, 1983.

12. Borer, K. T., M. Trulson and L. R. Kuhns. The role of limbic system in the control of hamster growth. Brain Res Bull 4: 239 $247,1979$.

13. Brown, G. M., D. S. Schalch and S. Reichlin. Patterns of growth hormone and cortisol responses to psychological stress in the squirrel monkey. Endocrinology 88: 956-963, 1971.
14. Brownstein, M., A. Arimura, H. Sato, A. V. Schally and J. S. Kizer. The regional distribution of somatostatin in the rat brain. Endocrinology 100: 246-249, 1977.

15. Critchlow, V., F. Abe, S. Urman and W. Vale. Effects of lesions of the periventricular nucleus of the preoptic-anterior hypothalamus on growth hormone and thyrotropin secretion and brain somatostatin. Brain Res 222: 267-276, 1981.

16. Duddleson, W. G., A. R. Midgley, Jr. and G. D. Niswender. Computer program sequence for analysis and summary of radioimmunoassay data. Comp Biomed Res 5: 205-217, 1972.

17. Elde, R. P. and J. A. Parsons. Immunocytochemical localization of somatostatin in cell bodies of the rat hypothalamus. Am J Anat 144: 541-548, 1975.

18. Epelbaum, J., J. O. Willoughby, P. Brazeau and J. B. Martin. Effect of brain lesions and hypothalamic deafferentation on somatostatin distribution in the rat brain. Endocrinology 101: 1495-1502, 1977.

19. Gibson, J. G., II, W. P. Murphy, Jr., W. A. Scheitlin and S. B. Rees. The influence of extracellular factors involved in the collection of blood in $A C D$ on maintenance of red cell viability during refrigerated storage. Am J Clin Pathol 26: 855-873, 1957.

20. Gibson, J. G., C. B. Gregory and L. N. Button. Citratephosphate-dextrose solution for preservation of human blood. A further report. Transfusion 1: 280-287, 1961.

21. Hökfelt, T., S. Efendic, G. Hellerstrom, O. Johansson, R. Luft and A. Arimura. Cellular localization of somatostatin in endocrine-like cells and neurons of the rat with special reference to the A-cells of the pancreatic islets and to the hypothalamus. Acta Endocrinol [Suppl] (Copenh) 200: 5-41, 1975.

22. Merchenthaler, I., S. Vigh, A. V. Schally and P. Petrusz. Immunocytochemical localization of growth-hormone-releasing fautor in rat hypothalamus. Endocrinology 114: 1082-1085, 1984.

23. Meyer, V. and E. Knobil. Growth hormone secretion in the unanesthetized rhesus monkey in response to noxious stimuli. Endocrinology 80: 163-171, 1967.

24. Mitchell, J. A., R. Smyrl, M. Hutchins, W. J. Schindler and V. Critchlow. Plasma growth hormone levels in rats with increased naso-anal lengths due to hypothalamic surgery. Neuroendocrinology 10: 31-45, 1972.

25. Palkovits, M., R. M. Kobayashi, M. Brown and W. Vale. Changes in hypothalamic, limbic and extrapyramidal somatostatin levels following various hypothalamic transections in the rat. Brain Res 195: 499-505, 1980. 
26. Plotsky, P. M. and W. Vale. Patterns of growth hormone releasing factor and somatostatin secretion into the hypophysial-portal circulation in the rat. Sitence $230: 461-463$, 1985.

27. Potter, C. D., K. T. Borer and R. J. Katz. Opiate-receptor blockade reduces voluntary running but not self-stimulation in hamsters. Pharmacol Biochem Behav 18: 217-223, 1983.

28. Rice, R. W., J, Kroning and V. Critchlow. Sex differences in the effects of surgical isolation of the medial basal hypothalamus on linear growth and plasma growth hormone levels in the rat, Endocrinology 98: 982-990, 1976.

29. Schalch, D. S. The influence of physical stress and exercise on growth hormone and insulin secretion in man. J Lab Clin Med 69: $256-269,1967$.

30. Schalch, D. S. and S. Reichlin. Plasma growth hormone concentration in the rat determined by radioimmunoassay: Influence of sex. pregnancy, lactation, anesthesia, hypophysectomy and extrasellar pituitary transplant. Endocrinology 79: 275-280. 1966.
31. Schalch, D. S. and S. Reichlin. Stress and growth hormone release. In: Growth Hormone. Int Congr Series 158, edited by A. Pecile and E. E. Mueller. Amsterdam: Fxcerpta Medica Foundation, 1968, pp. 211-225.

32. Schindler, W., M. O. Hutchins and E. J. Septimus. Growth hormone secretion and control in the mouse. Endocrinology 91: $483-490,1972$.

33. Terry, L. C., J, O. Willoughby, P. Brazeau, J. B. Martin and Y. Patel. Antiserum to somatostatin prevents stress-induced inhjbition of growth hormone secretion in the rat. Science 192: $565-567,1976$.

34. Thorell, J. L. and B. G. Johansson. Enzymatic iodination of polypeptides with ${ }^{125} \mathrm{I}$ to high specific activity. Biochim Biophys Acta 251: 363-369, 1971 . 